\title{
A CONJUGACY CRITERION
}

\author{
ROBERT R. STEVENS
}

\begin{abstract}
A sufficient condition is given for the differential equation $x^{\prime \prime}+p(t) x=0$ to have a nontrivial solution with more than one zero in a closed irterval $[a, b]$. A partial converse is obtained and applications are given.
\end{abstract}

1. Introduction. The differential equation

$$
x^{\prime \prime}+p(t) x=0
$$

is conjugate on a real interval $I$ if some nontrivial solution of (1) has more than one zero in $I$; otherwise, (1) is disconjugate on $I$. We shall assume throughour that $p(t)$ is continuous on the interval $I$.

Liapounoff [7] proved that equation (1) is disconjugate on the closed interval $[a, b]$ if $(b-a) \int_{a}^{b}|p(t)| d i \leqq 4$. This was generalized by Hartman and Wintner [5] who showed that the condition $\int_{a}^{b}(t-a)|p(t)| d t \leqq 1$ is also sufficient for disconjugacy on $[a, b]$, the latter theorem being derived from the following rephrased result of [5] which gives a necessary condition for conjugacy.

THEOREM. If $(1)$ is conjugate on $[a, b]$ and $p(t) \geqq 0$ then

$$
\int_{a}^{b}(t-a)(b-t) p(t) d t>b-a .
$$

It can be seen intuitively that if (1) is conjugate then $p(t)$ must be sufficiently large in some sense, as defined, for instance, by condition (2). However, (2) is not sufficient for conjugacy since, for example, the equation $x^{\prime \prime}+x=0$ is disconjugate on the interval $[0,3]$.

In [2], [3], [6] are given simpie criteria applicable to the equation $x^{\prime \prime}+(\lambda \sin t) x=0$. We mention also the fact that although Yelchin [9] states a sufficient condition, it is not explicit, and its usefulness in particular conjugacy problems for bounded intervals is difficult to determine.

The idea of this paper is to obtain a "largeness" condition similar to (2) and which is explicit and sufficient for conjugacy on a closed interval $[a, b]$ (Theorem 1). A similar condition is proved to be necessary for conjugacy on the half open interval $[a, b)$ (Theorem 2).

Received by the editors March 3, 1971 and, in revised form, July 20, 1971.

AMS 1970 subject classifications. Frimary 34C10; Secondary 34B05.

Key words anc' phrases. Conjugate differential equations, disconjugacy, oscillation, eigenvalue problems.

(c) American Mathematical Society 1972 
These results are applicable to specific conjugacy and oscillation problems and to the estimation of eigenvalues, as well as upper bounds for the interval of uniqueness, for two point boundary value problems. Our results show, for example, that

(i) the equation $x^{\prime \prime}+\lambda \sin t x=0$ is conjugate on $[0, \pi]$ if $\lambda \geqq \frac{5}{4}$; hence,

(ii) the equation $x^{\prime \prime}+p(t) x=0$ is oscillatory if $p(t)$ dominates $\frac{5}{4} \sin t$ $\left(p(t) \geqq \frac{3}{4} \sin t\right)$ on infinitely many of the intervals $[n \pi,(n+1) \pi](n=0,2$, $4, \cdots)$, and $\lambda<\frac{5}{4}$

(iii) the first eigenvalue of $x^{\prime \prime}+\lambda \sin t x=0 ; x(0)=0, x(\pi)=0$ satisfies

Applications such as that of (iii) are extremely useful when used in conjunction with the necessary condition of (2). For example, application of (2) to the example in (iii) shows that a lower bound for the first eigenvalue in 0.73 .

We wish to thank the referee for his helpful suggestions concerning the organization of the paper and also for the reference to [8] where it is proved that the equation in (i) above is conjugate for $\lambda \geqq 1.242$.

2. Main results. We shall suppose throughout that $p(t) \geqq 0$ and is continuous on a closed interval $[a, b]$. Let $T_{a, b}$ be the operator on $C[a, b]$, defined by

$$
T_{a, b}: f(t) \rightarrow \frac{b-t}{b-a} f(a)+\frac{t-a}{b-a} f(b)-\int_{a}^{b} G(t, s) p(s) f(s) d s,
$$

where $G(t, s)$ is the Green's function for $y^{\prime \prime}$ on $[a, b]$ :

and

$$
G(t, s)=\frac{(b-s)(t-a)}{a-b} \text { for } a \leqq t \leqq s
$$

$$
G(t, s)=\frac{(s-a)(b-t)}{a-b} \text { for } s \leqq t \leqq b .
$$

Note that, for $p \geqq 0$ on $[a, b], T_{a . b}$ is a monotone operator, $\left(T_{a . b} f\right)^{\prime \prime}=-p f$, and $T_{a, b} f$ and $f$ are equal at $a$ and $b$. The function sequences $R_{n}(t)$ and $L_{n}(t)$ are defined for $a \leqq t \leqq b$ by

$$
R_{n+1}(t)=T_{a, b}^{n}\left(\frac{b-t}{b-a}\right) \quad \text { and } \quad L_{n+1}(t)=T_{a . b}^{n}\left(\frac{t-a}{b-a}\right)
$$

for $n \geqq 0$. When context requires, we shall express the dependence on $a, b, p$ by writing $R_{n}(t ; p, a, b)$ and $L_{n}(t ; p, a, b)$.

THEOREM 1. Let $p(t) \geqq 0$ on $[a, b]$. If there exists $a<c<b$ and $m \geqq 1$ 
such that

and

$$
\int_{a}^{c} \int_{t}^{c} L_{m}(s ; p, a, c) p(s) d s d t \geqq 1
$$

$$
\int_{c}^{b} \int_{c}^{t} R_{m}(s ; p, c, b) p(s) d s d t \geqq 1
$$

then the equation (1) is conjugate on $[a, b]$.

EXAMPLE: A straightforward calculation for the equation

$$
x^{\prime \prime}+\lambda \sin t x=0
$$

on the interval $[0, \pi]$ yields (5) and (6), with $c=\pi / 2$ and $m=2$, when $\lambda \geqq \frac{5}{4}$. Thus (7) is conjugate on the interval $[0, \pi]$ when $\lambda \geqq \frac{5}{4}$. Note also that statements (ii) and (iii) of the introduction follow from this, through use of Sturm's theorem. As a partial converse to Theorem 1, we have

THEORFM 2. Let $p(t) \geqq 0$ on $[a, b]$ and let $p(t)$ not vanish identically in any subinterval of $[a, b]$. If equation (1) is conjugate on the half open interval $[a, b)$, then there exists $a<c<b$ and $m \geqq 1$ such that strict inequality ( $>$ ) hoids in both (5) and (6) of the statement of Theorem 1.

We omit the statements of the equivalent forms of Theorem 2 which give sufficient (although not explicit) conditions for disconjugacy on the half open interval $[a, b)$.

COROLLARY 1. Let $p(t) \geqq 0$ on $[a, b]$, and let $x$ be the solution of equation (1) satisfying $x^{\prime}(a)=0$ and $x(a)=1$.

(i) If there exists $m \geqq 1$ such that $\int_{a}^{b} \int_{a}^{t} R_{m}(s) p(s) d s d t \geqq 1$, then $x$ vanishes at some point of $[a, b]$.

(ii) If $x$ vanishes at some point of $[a, b)$, then there exists $m \geqq 1$ such that $\int_{a}^{b} \int_{a}^{t} R_{m}(s) p(s) d s d t>1$.

COROLlaRY 2 . Let $p(t) \geqq 0$ on $[a, b]$ and let $x$ be the solution of equation (1) satisfying $x(a)=0, x^{\prime}(a)=1$.

(i) If there exists $m \geqq 1$ such that $\int_{a}^{b} \int_{a}^{b} L_{m}(s) p(s) d s d t \geqq 1$, then $x^{\prime}$ vanishes at some point of $[a, b]$.

(ii) If $x^{\prime}$ vanishes at some point of $[a, b]$ and $p$ does not vanish identically on any subinterval of $[a, b]$, then there exists $m \geqq 1$ such that

$$
\int_{a}^{b} \int_{t}^{b} L_{m}(s) p(s) d s d t>1
$$

\section{Proofs of the theorems.}

Proof OF ThForem 1. Define $R_{n}(t)=R_{n}(t ; p, c, b)$ and $L_{n}(t)=$ $L_{n}(t ; p, a, c)$ (see (4)). Since $\left(R_{2}-R_{1}\right)^{\prime \prime} \leqq 0$ and $R_{2}=R_{1}$ at $c$ and $b$, it 
follows that $R_{2} \geqq R_{1}$. Hence by the monotonicity of the operator $T_{c, b}$, $\left\{R_{n}\right\}$ is nondecreasing. By the same argument, $\left\{L_{n}\right\}$ is nondecreasing. Let $x(t)$ satisfy equation (1) and $x(a)=0, x^{\prime}(a)=1$. If $x^{\prime}>0$ on $[a, c]$, then $x>0$ on $(a, c]$, and $y=x / x(c)$ satisfies equation (1) and $y(a)=0$, $y(c)=1$. Since $\left(T_{a, c} y\right)^{\prime \prime}=-p y=y^{\prime \prime}$ and $T_{a, c} y$ and $y$ are equal at $a$ and $c$, it follow's that $y=T_{a, c} y$ on $[a, c]$. Clearly, $\left(y-L_{1}\right)^{\prime \prime} \leqq 0$ and $y=L_{1}$ at $c$ and $b$, so that $y \geqq L_{1}$. By monotonicity of $T_{a, c}, y \geqq L_{n}$ for all $n$. Since $-y^{\prime \prime}=$ $p y \geqq p L_{m}, y^{\prime}(c)-y^{\prime}(t)=\int_{t}^{c} y^{\prime \prime}(s) d s \leqq-\int_{t}^{c} p(s) L_{m}(s) d s$. Another integration yields

or

$$
\int_{a}^{c}\left[y^{\prime}(c)-y^{\prime}(t)\right] d t \leqq-\int_{a}^{c} \int_{t}^{c} p(s) L_{m}(s) d s
$$

Thus

$$
-y^{\prime}(c)(c-a)+[y(c)-y(a)] \geqq \int_{a}^{c} \int_{t}^{c} p(s) L_{m}(s) d s .
$$

$$
\int_{a}^{c} \int_{t}^{c} p(s) L_{m}(s) d s \leqq-y^{\prime}(c)(c-a)+1<1 .
$$

This contradicts (5) and shows that $x^{\prime}$ cannot be positive everywhere in $[a, c]$.

Now suppose that $x$ (defined above) satisfies $x>0$ on $(a, b]$. Then $x^{\prime \prime} \leqq 0$, and thus $x^{\prime}(c) \leqq 0$. Since $y \geqq R_{1}$ at the endpoints of $[c, b]$ and $\left(y-R_{1}\right)^{\prime \prime}=y^{\prime \prime} \leqq 0$, it follows that $y \geqq R_{1}$. As above, $y=T_{c, b} y$ on $[c, b]$ and by monotonicity, $y \geqq R_{n}$ for all $n$. Since $-y^{\prime \prime}=p y \geqq p R_{m}, y^{\prime}(t)-y^{\prime}(c)=$ $\int_{c}^{t} y^{\prime \prime}(s) d s \leqq-\int_{c}^{t} p(s) R_{m}(s) d s$. Another integration gives

or

$$
\int_{c}^{b}\left[y^{\prime}(t)-y^{\prime}(c)\right] d t \leqq-\int_{c}^{b} \int_{c}^{t} p(s) R_{m}(s) d s d t,
$$

$$
\begin{aligned}
\int_{c}^{b} \int_{c}^{t} p(s) R_{m}(s) d s d t & \leqq y^{\prime}(c)(b-c)-[y(b)-y(c)] \\
& \leqq y(c)-y(b)<y(c)=1 .
\end{aligned}
$$

This contradicts (6) and shows, finally, that $x$ vanishes at some point of $(a, b]$. This proves that equation (1) is conjugate on $[a, b]$.

Proof of Theorem 2. The hypothesis implies that the solution $x$ of equation (1) satisfying $x(a)=0, x^{\prime}(a)=1$ has a zero in the open interval $(a, b)$. Let $z$ be the smallest such zero, $a<z<b$. Then $x>0$ in $(a, z)$ and there exists $d, a<d<z$, such that $x^{\prime}(d)=0, x^{\prime}>0$ in $[a, d)$ and $x(d)>0$. Suppose now that

$$
\int_{d}^{b} \int_{d}^{t} R_{n}(s ; p, d, b) p(s) d s d t \leqq 1 \quad(n \geqq 1) .
$$


Recall that $\left\{R_{n}\right\}$ is nondecreasing on $[d, b]$. Also from (4) and (8),

$$
\begin{aligned}
R_{n+1}(t) & =\left(T_{d, b} R_{n}\right)(t) \\
& =\frac{b-t}{b-d}+\frac{t-d}{b-d} \int_{d}^{b} \int_{a}^{s} p(u) R_{n}(u) d u d s-\int_{d}^{t} \int_{d}^{s} p(u) R_{n}(u) d u d s \\
& \leqq \frac{b-t}{b-d}+\frac{t-d}{b-d}=1
\end{aligned}
$$

for all $n \geqq 1$. Let $R_{n} \rightarrow R$. By the monotone convergence theorem of the Lebesgue theory and (4), $R=T_{d, b} R$. Hence $R^{\prime \prime}=\left(T_{i, b} R\right)^{\prime \prime}=-p R$ so that $R$ is a solution of equation (1). Since $R_{1}(d)=1$ and $R_{1}(b)=0$, and the operator $T_{d, b}$ preserves these boundary values, $R(d)=1$ and $R(b)=0$. Also,

$$
R^{\prime}(d)=\frac{-1}{b-d}+\frac{1}{b-d} \int_{d}^{b} \int_{d}^{s} p(u) R(u) d u d s \leqq 0,
$$

the last inequality being implied by $(8)$. Note that actually $R^{\prime}(d)<0$. (If $R^{\prime}(d)=0$, then $R$ would be a multiple of $x$, since $x^{\prime}(d)=0$, and hence would vanish at $z$. But $R(z) \geqq R_{1}(z)=(b-z) /(b-d)>0$.) Since $R \geqq R_{1}$, $R>0$ on $[d, z]$. Thus $y=R-x / x(d)$ satisfies $(1), y(d)=0, y(z)>0$, and (since $\left.y^{\prime}(d)<0\right) y(t)<0$ for $t$ near $d$. Hence there exists $w \in(d, z)$ such that $y(w)=0$. Since $y(d)$ is also zero, Sturm's theorem implies that $R$ vanishes at some point in $[d, w]$. However, this contradicts $R>0$ on $[d, z]$ and shows that (8) cannot hold. Hence there exists $k \geqq 1$ such that $\int_{d}^{b} \int_{d}^{t} R_{k}(s ; p, d, b) p(s) d s d t>1$ and, by continuity, there exists $c \in(d, z)$ such that

$$
\int_{c}^{b} \int_{c}^{t} R_{k}(s ; p, c, b) p(s) d s d t>1 .
$$

Consider now the interval $[a, c]$ and recall that $x^{\prime}(d)=0$, where $a<d<c$, and that $x^{\prime}>0$ in $[a, d)$. Suppose now that

$$
\int_{a}^{c} \int_{t}^{c} L_{n}(s ; p, a, c) p(s) d s d t \leqq 1 \quad(n \geqq 1) .
$$

Using the same argument as above for $\left\{R_{n}\right\}$, it follows that there exists $L$ which is a solution of (1) on $[a, c]$ and such that $L(a)=0, L(c)=1$ and $L^{\prime}(c) \geqq 0$. Since $p$ does not vanish identically in $[d, c]$, it follows that

$$
L^{\prime}(d)=L^{\prime}(c)-\int_{d}^{c} L^{\prime \prime}(s) d s \geqq \int_{d}^{c} p(s) L(s) d s>0 \quad \text { (strict inequality). }
$$

Hence $L^{\prime}(d)>0$. However, since the solutions $L$ and $x$ both vanish at $a$, they are linearly dependent. This implies, since $x^{\prime}(d)=0$, that $L^{\prime}(d)=0$. 
This contradiction shows that (10) cannot hold. Hence there is an in teger $n \geqq 1$ such that

$$
\int_{a}^{c} \int_{t}^{c} L_{n}(s ; p, a, c) p(s) d s d t>1 .
$$

By (9) and (11), letting $m=\max \{k, n\}, c$ and $m$ are as desired. The proof is complete.

The proofs of the corollaries are similar and are omitted.

\section{REFERENCES}

1. Dallas Banks, Upper bounds for the eigenvalues of some vibrating systems, Pacific J. Math. 11 (1961), 1183-1203. MR 26 \#2681.

2. A. M. Fink, The functional $T \int_{0}^{T} R$ and the zeroes of a second order linear differential equation, J. Math. Pures Appl. (9) 45 (1966), 387-394. MR 34 \#7863.

3. A. S. Galbraith, On the zeros of solutions of ordinary differential equations of the second order, Proc. Amer. Math. Soc. 17 (1966), 333-337. MR 32 \#7848.

4. P. Hartman, Ordinary differential equations, Wiley, New York, 1964. MR 30 \#1270.

5. P. Hartman and A. Wintner, On an oscillation criterion of Liapounoff, Amer. J. Math. 73 (1951), 885-890. MR 13, 652.

6. Walter Leighton, On the zeros of solutions of a second-order linear differential equation, J. Math. Pures Appl. (9) 44 (1965), 297-310. MR 32 \#4323.

7. A. Liapounoff, Sur une série relative à la théorie des équations différentielles linéaires à coefficient périodiques, C.R. Acad. Sci. Paris 123 (1896), 1248-1252.

8. D. Willett, Oscillation on finite or infinite intervals of second order linear differential equations, Canad. Math. Bull. (to appear).

9. M. Yelchin, Sur les conditions pour qu'une solution d'un système linéaire du second ordre possède deux zéros, C.R. (Dokl.) Acad. Sci. URSS 51 (1946), 573-576. MR 8, 208.

Department of Mathematics, loyola University, New Orleans, Louisiana, 70118

Department of Mathematics, University of Montana, Missoula, Montana 59801 